08,04

\title{
Применение высокочастотного ЭПР/ЭСЭ для идентификации примесного состава и электронной структуры керамик на основе гранатов
}

\author{
(C) Е.В. Единач, Ю.А. Успенская, А.С. Гурин, Р.А. Бабунц, Г.Р. Асатрян, Н.Г. Романов, \\ А.Г. Бадалян, П.Г. Баранов \\ Физико-технический институт им. А.Ф. Иоффре РАН, \\ Санкт-Петербург, Россия \\ E-mail: elena.edinach@mail.ioffe.ru
}

Поступила в Редакцию 3 июня 2019 г.

В окончательной редакции 3 июня 2019 г.

Принята к публикации 4 июня 2019 г.

Обнаружены и идентифицированы спектры электронного парамагнитного резонанса (ЭПР) примесных ионов $\mathrm{Ce}^{3+}, \mathrm{Yb}^{3+}, \mathrm{Cr}^{3+}$ и $\mathrm{Gd}^{3+}$ в керамиках иттрий-алюминиевого граната $\mathrm{Y}_{3} \mathrm{Al}_{5} \mathrm{O}_{12}$ (YAG) на частоте $94 \mathrm{GHz}$. Показано преимущество измерения спектров ЭПР в высокочастотном диапазоне по сравнению со стандартной техникой ЭПР, позволяющее разделить спектры ЭПР, характеризующиеся разными анизотропными $g$-факторами, а также выделить сигналы ЭПР, обусловленные расщеплением тонкой структуры для центров с высоко-спиновыми состояниями. В керамиках с высоким содержанием магнитных ионов $\mathrm{Gd}^{3+}$ наблюдались спектры ЭПР и электронного спинового эха (ЭСЭ) много-ионных комплексов гадолиния, при этом при низких температурах видны спектры ЭПР комплексов с максимальным количеством обменносвязанных ионов гадолиния. Исследования температурных зависимостей спектров ЭПР свидетельствует о ферромагнитном упорядочении обменно-связанных комплексов гадолиния.

Ключевые слова: электронный парамагнитный резонанс, электронное спиновое эхо, гранаты, керамика, редкоземельные ионы, переходные ионы.

DOI: 10.21883/FTT.2019.10.48262.503

\section{1. Введение}

Кристаллы гранатов с примесью излучающих редкоземельных элементов и керамики на их основе являются уникальными системами как для фундаментальных исследований, так и для многочисленных применений.

Решающее значение при создании сцинтилляторов для регистрации ионизирующего излучения, например, при создании сцинтилляторов для позитронно-эмиссионной или компьютерной томографии, оптимизации их параметров играет выбор материалов для сцинтилляционных детекторов, т.е. веществ, обладающих способностью излучать свет при поглощении ионизирующего излучения [1-4]. Кристаллы и керамики гранатов с примесью церия широко используются для преобразования света голубых фотодиодов и лазеров на основе нитридов в белый свет. Оптимизация свойств этих материалов также является актуальной задачей [5-6].

Имеется ряд требований к временным характеристикам сцинтилляторов, например, низкий уровень послесвечения, отсутствие вторичных компонентов распада в сцинтилляционной вспышке [7]. Эти явления обусловлены наличием центров захвата в виде собственных и примесных дефектов в кристалле, при этом при комнатной температуре глубокие ловушки ответственны за послесвечение, а время затухания сцинтилляции связано с тепловым выделением носителей из мелких ловушек.
Новое направление в использовании гранатов с примесью излучающих редкоземельных ионов появилось в последнее время в связи с возможностью их потенциального использования в квантовых вычислениях и квантовых коммуникациях [8-10]. Кристаллы с примесью редкоземельных ионов являются идеальными излучателями и, одновременно, спиновыми системами как электронными, так и ядерными. Недавно оптически был обнаружен магнитный резонанс на одиночном ионе $\mathrm{Ce}^{3+}$ в иттрий-алюминиевом гранате. Такие системы являются модельными для осуществления взаимодействия между одиночными фотонами (оптическими кубитами) и одиночными спинами (спиновыми кубитами), что составляет основу для осуществления квантовых манипуляций при квантовых вычислениях и коммуникациях.

Электронный парамагнитный резонанс (ЭПР) и электронное спиновое эхо (ЭСЭ) являются наиболее прямыми методами исследования переходных и редкоземельных элементов в конденсированных материалах Основным направлением в современных ЭПР исследованиях является повышение чувствительности и спектрального разрешения спектрометров путем увеличения рабочей частоты. Повышение рабочей частоты также позволяет проводить регистрацию спектров ЭПР для систем с высокими спиновыми состояниями, включая многочастичные парамагнитные комплексы, значительные рас- 
щепления тонкой структуры и обменные расщепления которых, как правило, не позволяют наблюдать спектры ЭПР на традиционных частотах. При этом принципиальную роль играет также возможность понижения рабочей температуры, что позволяет кроме повышения чувствительности, устанавливать порядок расположения энергетических уровней, то есть определять знаки параметров тонкой структуры и обменных взаимодействий.

В работе для изучения электронной структуры примесей в нескольких типах керамик был использован разработанный в ФТИ им. А.Ф. Иоффе в рамках ФЦП Минобрнауки радиоспектроскопический комплекс, включающий новую методику регистрации ЭПР и ЭСЭ в ближнем терагерцовом диапазоне с использованием магнито-оптического криостата замкнутого цикла. В настоящей работе были разделены сигналы ЭПР различных примесей переходных и редкоземельных металлов благодаря специфическим особенностям в зависимостях этих сигналов от магнитного поля и температуры. Обнаружены спектры ЭПР многочастичных парамагнитных комплексов из ионов гадолиния в керамиках на основе лютециевых гранатов с высокой концентрацией гадолиния, замещающего диамагнитные ионы лютеция.

\section{2. Методика эксперимента}

Методика изготовления образцов. Иттрий-алюминиевые гранаты $\mathrm{Y}_{3} \mathrm{Al}_{5} \mathrm{O}_{12}$ (YAG) кристаллизуются в виде кубической решетки и описываются формулой $\mathrm{C}_{3} \mathrm{~A}_{2} \mathrm{D}_{3} \mathrm{O}_{12}$, которую можно также записать в следующей форме: $\mathrm{C}_{3}(\mathrm{~A}, \mathrm{D})_{5} \mathrm{O}_{12}$, где обозначены три различных катионных позиции: с - додекаэдрическая позиция, a и d - октаэдрическая и тетраэдрическая позиции. Ионы иттрия занимают додекаэдрические позиции (c), ионы алюминия занимают октаэдрические (а) и тетраэдрические (d) позиции. Существует только один тип ионов $\mathrm{O}^{2-}$, тетраэдрически связанный с двумя ионами $\mathrm{A}^{3+}$ и двумя ионами $\mathrm{Y}^{3+}$.

Керамики YAG: Ce,Yb; YAG: Ce,Cr; YAG: Ce,Yb,Gd и $(\mathrm{Gd}, \mathrm{Lu})_{3}(\mathrm{Al}, \mathrm{Ga})_{5} \mathrm{O}_{12}$ были синтезированы по методике, описанной ранее в работах [7,11-13]. Керамики были получены путем смешивания порошков исходных оксидов $\mathrm{Y}_{2} \mathrm{O}_{3}, \mathrm{Lu}_{2} \mathrm{O}_{3}, \mathrm{Gd}_{2} \mathrm{O}_{3}, \mathrm{Al}_{2} \mathrm{O}_{3}$ и $\mathrm{Ga}_{2} \mathrm{O}_{3}$ с добавлением оксидов соответствующих примесей с концентрацией примерно $0.1 \%$, добавлением связующих органических материалов; прессованием и термообработкой для выжигания органических материалов. Затем отожженные спрессованные формы подвергались спеканию, температура синтеза подбиралась в зависимости от композиции для получения единой фазы граната. По данным электронной и конфокальной оптической микроскопии средний размер зерна составлял $5-8 \mu \mathrm{m}$.

Было показано, что примесь гадолиния изменяет зонную картину граната и приводит к улучшению характеристик сцинтилляторов. Методы ЭПР позволяют де- тально исследовать взаимодействие между магнитными ионами гадолиния $\mathrm{Gd}^{3+}$ при увеличении их концентрации. При этом иттрий $\mathrm{Y}^{3+}$ и лютеций $\mathrm{Lu}^{3+}$ являются диамагнитными ионами, и метод ЭПР не может быть использован для их исследования.

В настоящей работе были также исследованы керамики с повышенным содержанием магнитной примеси гадолиния $\mathrm{Gd}_{2.7} \mathrm{Lu}_{0.3} \mathrm{Ga}_{2.5} \mathrm{Al}_{2.5} \mathrm{O}_{12}: \mathrm{Ce}(0.4 \%)$, поскольку такие керамики часто используются при создании сцинтилляторов.

На рис. 1 представлена упрощенная схема спектрометра ЭПР/ЭСЭ, используемого в наших экспериментах и работающего на длинах волн 3 и $2 \mathrm{~mm}$ в едином приборе.

Достоинством такой схемы является минимизация отраженного от различных неоднородностей микроволнового сигнала, обычно проявляющегося в стандартных схемах, использующих микроволновый резонатор и транспортировку микроволновой мощности 3 или $2 \mathrm{~mm}$ диапазонов по волноводам большего сечения. При этом размеры образца ограничены диаметрами кварцевых трубок с диаметрами 0.5 и $0.3 \mathrm{~mm}$, соответственно.

Спектрометр содержит микроволновый блок (рис. 1), который включает высокостабильный твердотельный генератор фиксированной частоты $f=7.23 \mathrm{GHz}$, сигнал с которого поступает на вход умножителя частоты на $N$ основного генератора и на $N-1$ гетеродина. Сигнал с выхода основного генератора через усилитель, аттенюатор и первый модулятор поступает на вход циркулятора, вход/выход которого соединяется с прямоугольным волноводом используемого диапазона СВЧ - 3 или $2 \mathrm{~mm}$. Прямоугольный волновод через специальный переход соединяется с цилиндрическим волноводом, который помещается в рабочий объем криостата и заканчивается цилиндрическим стаканом того же диаметра, что и цилиндрический волновод, служащий резонатором без верхней крышки. На дно упомянутого стакана помещается исследуемый образец. Диаметр цилиндрического волновода выбирается исходя из условий оптимального прохождения микроволновой волны простейшей конфигурации $\mathrm{TE}_{01}$. Вращение образца в магнитном поле осуществляется путем вращения цилиндрического волновода.

Высокочастотный сигнал поступает на образец, а отраженный сигнал через циркулятор и второй модулятор поступает на смеситель приемника, и после усилителя промежуточной частоты - на квадратурный (IQ) детектор. Используется коэффициент $N=13$ для $3 \mathrm{~mm}$ диапазона и $N=18$ для $2 \mathrm{~mm}$ диапазона, то есть микроволновый блок построен на общей технологической схеме для 3 и $2 \mathrm{~mm}$ диапазонов. Первый модулятор передатчика и второй модулятор приемника необходимы для работы спектрометра в импульсном режиме, например, при регистрации электронного спинового эха. Работой модуляторов управляет формирователь импульсов, который создает необходимую последовательность импульсов и запрещает включение приемника 


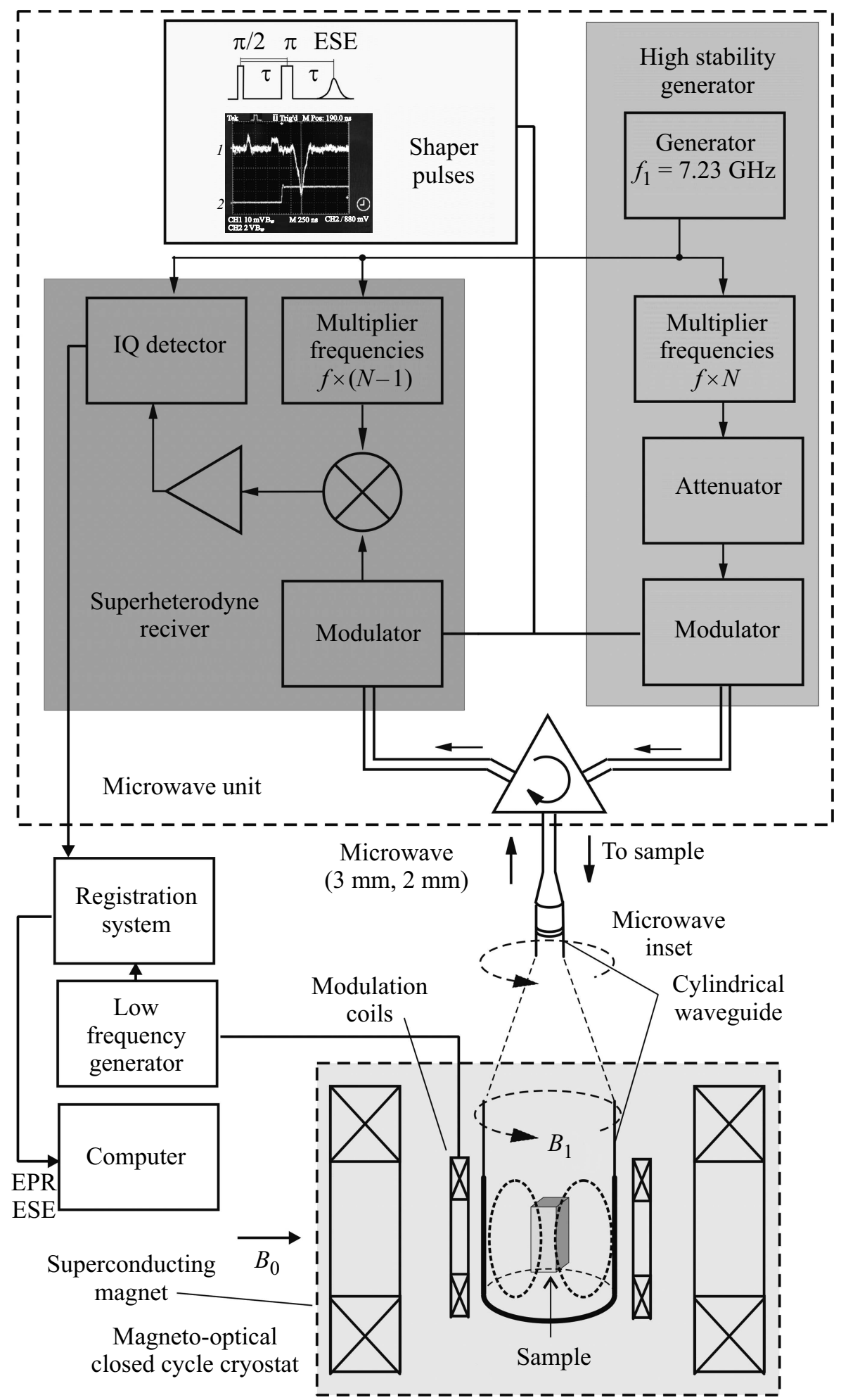

Pис. 1. Схема высокочастотного спектрометра ЭПР/ЭСЭ. Названия входящих в спектрометр блоков указаны на рисунке.

при включенном передатчике. В предложенном примере длительность импульса (от $10 \mathrm{~ns}$ до $0.1 \mathrm{~s}$ ) задается с шагом $3.2 \mathrm{~ns}$.

В спектрометре используется магнито-оптический криостат замкнутого цикла со сверхпроводящим магни- том, с возможностью развертки магнитного поля от -7 до $+7 \mathrm{~T}$, с плавным переходом через нулевое значение магнитного поля. Микроволновая вставка помещается в рабочий объем криостата, позволяющий изменять температуру на образце в диапазоне $1.5-300 \mathrm{~K}$. 


\section{3. Результаты и обсуждение результатов}

Спиновый гамильтониан, описывающий различные взаимодействия в парамагнитном центре, может быть записан в следующем виде

$$
\hat{H}=\mu_{B} \mathbf{B} \cdot \mathbf{g} \cdot \hat{\mathbf{S}}+\hat{\mathbf{S}} \cdot \mathbf{D} \cdot \hat{\mathbf{S}}+\hat{\mathbf{S}} \cdot \mathbf{A} \cdot \hat{\mathbf{I}}-g_{I} \boldsymbol{\mu}_{N} \mathbf{B} \cdot \hat{\mathbf{I}}+\hat{\mathbf{I}} \cdot \mathbf{Q} \cdot \hat{\mathbf{I}},
$$

где первое слагаемое описывает анизотропное зеемановское взаимодействие, второе слагаемое описывает взаимодействие тонкой структуры (для систем со спином $S>1 / 2$ ), третье слагаемое описывает анизотропное сверхтонкое взаимодействие. Последние два слагаемых описывают зеемановское ядерное взаимодействие и квадрупольное ядерное взаимодействие, которые важны для описания экспериментов по двойному электронноядерному резонансу и, как правило, не проявляются непосредственно в спектрах ЭПР. $\mu_{B}$ и $\mu_{N}$ магнетон Бора и ядерный магнетон, соответственно.

Важно отметить, что только зеемановские взаимодействия включают магнитное поле, остальные слагаемые не содержат магнитное поле. Таким образом, эксперименты на разных рабочих частотах позволяют разделить взаимодействия, зависящие и не зависящие от магнитного поля, поскольку резонансное магнитное поле определяется рабочей частотой спектрометра.

Минимально регистрируемая концентрация спинов $N_{\min }$ (для образцов малых размеров, которые не могут быть увеличены): $N_{\min } \propto \omega^{-9 / 2}$, где $\omega=2 \pi \nu$, где $v-$ частота спектрометра ЭПР.

Спектральное разрешение определяется возможностью регистрировать малые изменения $g$-фактора $\Delta g$, которые могут быть записаны в виде $\Delta B=-\Delta g B / g$, где $\Delta B$ - изменение положения линии ЭПР в магнитном поле (сдвиг линии ЭПР) при изменении $g$-фактора $\Delta g$, и это изменение пропорционально величине магнитного поля $B$, которое пропорционально частоте. Рабочая частота $v$ связана с резонансным магнитным полем $B$ для простейшей системы со спином $S=1 / 2$ и изотропным электронным $g$-фактором соотношением $h v=g \mu_{B} B$.

Таким образом, увеличение рабочей частоты спектрометра от традиционной частоты 9.4 до $94 \mathrm{GHz}$ приводит к увеличению разрешающей способности спектрометра в 10 раз, а последующее увеличение частоты до $130 \mathrm{GHz}$ приводит к дальнейшему увеличению разрешающей способности спектрометра еще в 1.4 раза. При этом чувствительность увеличится в первом случае в $(10)^{9 / 2} \approx 30000$ раз, а во втором еще в $(1.4)^{9 / 2} \approx 4.5$ раза. Увеличение рабочей частоты спектрометра ЭПР также приводит к достижению более высоких больцмановских факторов, играющих определяющую роль во многих физических спинзависимых процессах, включая динамическую поляризацию ядер.

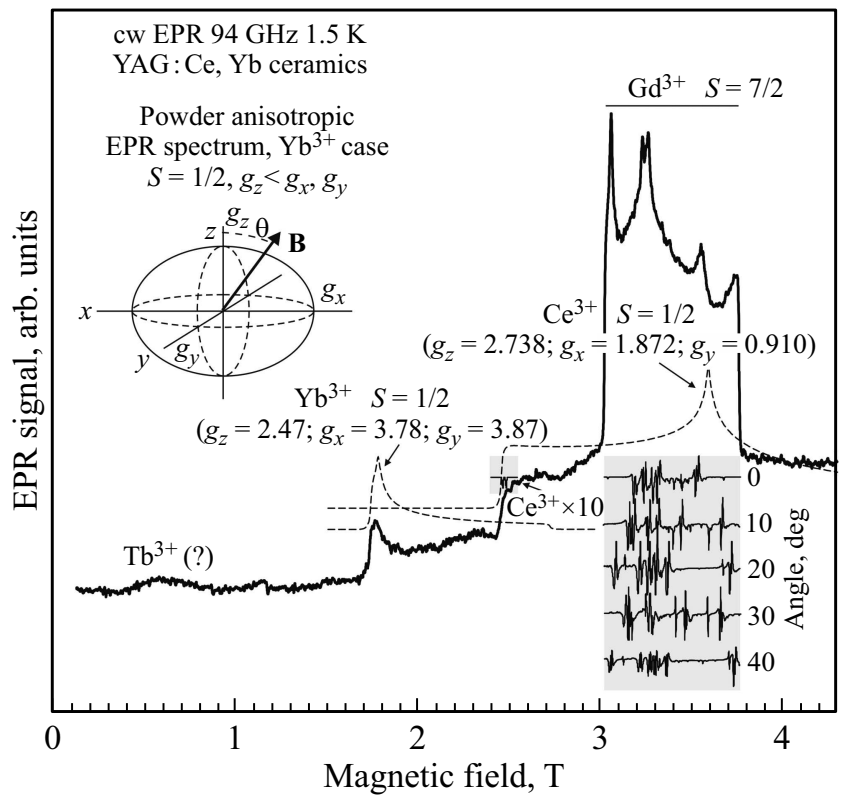

Рис. 2. Спектр ЭПР, зарегистрированный в керамике YAG:Ce,Yb. Результаты симуляции спектров ЭПР $\mathrm{Yb}^{3+}$ и $\mathrm{Ce}^{3+}$ в порошковом материале показаны в виде пунктирной линии. На вставке приведены спектры ЭПР для ионов $\mathrm{Gd}^{3+}$ в монокристалле YAG: Ce, Gd, зарегистрированные в нескольких ориентациях.

Для образцов в виде порошковых материалов или керамик, представляющих собой набор случайно ориентированных в пространстве микрокристаллов, спектры ЭПР имеют ряд особенностей, обусловленных случайной ориентацией этих микрокристаллов в магнитном поле. Естественно, речь идет о парамагнитных центрах, характеризующихся анизотропными параметрами спинового гамильтониана (1). Например, для парамагнитных центров со спином $S=1 / 2$ и анизотропным $g$-фактором (именно такими центрами являются ионы $\mathrm{Ce}^{3+}$ и $\mathrm{Yb}^{3+}$, исследованные в настоящей работе) в заданном магнитном поле $B$ для каждого парамагнитного центра должно выполняться резонансное условие $B=h v /\left[g(\theta) \mu_{B}\right]$, где $\theta$ - угол между направлением магнитного поля и осью $z$.

На рис. 2 показан спектр ЭПР в керамике YAG: Ce,Yb, зарегистрированный в непрерывном режиме на частоте $94 \mathrm{GHz}$ при температуре $1.5 \mathrm{~K}$. Указаны сигналы ЭПР, принадлежащие ионам $\mathrm{Ce}^{3+}$ и $\mathrm{Yb}^{3+}$.

Ион $\mathrm{Ce}^{3+}\left(4 f^{1}\right)$ имеет только один $4 f$-электрон. Конфигурация $4 f^{1}$ основного состояния дает два подтерма: нижний ${ }^{2} F_{5 / 2}$ и верхний ${ }^{2} F_{7 / 2}$, которые разделены в YAG энергетическим интервалом примерно $2000 \mathrm{~cm}^{-1}$. В кристаллическом поле для ионов $\mathrm{Ce}^{3+}$ в YAG имеется три крамерсовых дублета, энергии второго и третьего крамерсовых дублетов подтерма ${ }^{2} F_{5 / 2}$ отстоят от основного состояния на 228 и $587 \mathrm{~cm}^{-1}$, соответственно, и, как следствие, ЭПР-переходы наблюдаются только между компонентами самого низкого дублета, который заселя- 
ется только при температурах ниже $20 \mathrm{~K}$. Церий имеет только четные изотопы с нулевым ядерным магнитным моментом $(I=0)$. Спектры ЭПР и их угловые зависимости можно описать эффективным спином $S=1 / 2$ и анизотропным $g$-фактором со значениями $g_{Z}=2.74$, $g_{X}=1.87, g_{Y}=0.91[14,15]$ с использованием первого слагаемого спинового гамильтониана орторомбической симметрии (1). В монокристаллах YAG редкоземельные ионы занимают, как правило, додекаэдрические позиции кристаллической решетки (с-позиции), замещая, таким образом, ионы $\mathrm{Y}^{3+}$. В этом положении ионы $\mathrm{Y}^{3+}$ координируются восемью ионами кислорода с локальной симметрией D2 и образуют шесть магнитнонеэквивалентных центров $\mathrm{Ce}^{3+}$. Ось $z$ выбрана вдоль направления $\langle 100\rangle$ кристалла, оси $x$ и $y$ совпадают с направлениями $\langle 110\rangle$ в перпендикулярной оси $z$ плоскости.

Как и все ионы редкоземельных элементов, иттербий в YAG замещает иттрий в додекаэдрических позициях в трехвалентном зарядовом состоянии $\mathrm{Yb}^{3+}\left(4 f^{13}\right)$. С конфигурацией электронов $4 f^{13}$ (один отсутствующий электрон в заполненной $4 f$-оболочке) $\mathrm{Yb}$ является ,дырочным эквивалентом“ $\mathrm{Ce}^{3+}$ с конфигурацией электронов $4 f^{1}$ (один электрон в пустой $4 f$-оболочке). Оба иона характеризуются одинаковым термом ${ }^{2} F$, но с противоположным порядком расположения подтермов ${ }^{2} F_{5 / 2}$ и ${ }^{2} F_{7 / 2}$, образующихся в результате спин-орбитального взаимодействия с противоположным знаком параметра спин-орбитальной связи $\lambda$. При этом абсолютная величина спин-орбитального взаимодействия у иттербия с атомным номером $Z=70$ существенно больше аналогичной величины для церия с $Z=58$ [16], что приводит к значительному расщеплению подтермов для иттербия $\mathrm{Yb}^{3+}$, расстояние между двумя подтермами составляет примерно $10000 \mathrm{~cm}^{-1}$, при этом ${ }^{2} F_{7 / 2}$ является нижним мультиплетом в соответствие со вторым правилом Хунда. В результате переходы ${ }^{2} F_{5 / 2} \rightarrow{ }^{2} F_{7 / 2}$ в основном состоянии ${ }^{2} F[17,18]$ наблюдаются в ближней инфракрасной области спектра.

Оптические переходы между уровнями основного состояния ${ }^{2} F \mathrm{Yb}^{3+}$ в YAG хорошо изучены и надежно идентифицированы $[19,20]$. В частности, переход $1.03 \mu \mathrm{m}$ интересен для применения в мощных лазерах [21] и для реализации светодиодов с ИК-излучением и люминесценцией. Изменение постоянной решетки YAG : Се и, следовательно, свойств кристаллического поля в местах расположения центров церия путем замены иттрия иттербием в YAG:Cе может быть интересным для точной регулировки длины волны излучения церия.

Предполагается, что в порошках и керамиках гранатов с примесью ионов $\mathrm{Ce}^{3+}\left(4 f^{1}\right)$ и $\mathrm{Yb}^{3+}\left(4 f^{13}\right)$ образуются диамагнитные пары, состоящие из церия в четырехвалентном зарядовом состоянии $\mathrm{Ce}^{4+}\left(4 f^{0}\right)$ и иттербия в двухвалентном зарядовом состоянии $\mathrm{Yb}^{2+}\left(4 f^{14}\right)$. Этим объясняется резкое падение люминесценции церия $\mathrm{Ce}^{3+}$ при кодопировании гранатов церием и иттербием.

На вставке рис. 2 слева приведен слегка искаженный эллипсоид, демонстрирующий принцип усреднения сигнала ЭПР для $\mathrm{Yb}^{3+}$ с анизотропным $g$-фактором для порошкового материала при $g_{Z}<g_{X}, g_{Y}$ (так как $g_{X} \approx g_{Y}=g_{\perp}$, тогда $\left.g_{Z}=g_{\|}\right)$и выполняется условие $\left.g_{\|}<g_{\perp}\right)$. Результат симуляции спектра ЭПР $\mathrm{Yb}^{3+}$ в порошковом материале показан в виде пунктирной линии. Использованы величины $g$-факторов, полученные в работе [22] для ионов $\mathrm{Yb}^{3+}$ в кристалле $\mathrm{YAG:Yb.}$ $g_{Z}=2.47, g_{X}=3.78, g_{Y}=3.87$.

В результате усреднения по всем ориентациям микрокристаллов в магнитном поле наблюдается асимметричная форма линии ЭПР из-за того, что число спиновых пакетов, вносящих вклад в спектр, намного больше в плоскости $x y$, чем вдоль оси $z$. На вставке к рис. 2 аксиальный $g$-фактор с $g_{\perp}>g_{\|}$представлен в виде эллипсоида, и форма линии соответствующего ЭПР спектра показана в приближении большого числа парамагнитных центров в разных микрокристаллах с произвольной ориентацией их кристаллических осей по отношению к магнитному полю $B$. Отчетливо проявляются резонансные линии, которые коррелируют с экстремальными значениями угловой зависимости парамагнитных центров в монокристалле. Крайние позиции линий ЭПР порошкового спектра получены путем подстановки значений $g_{\|}$ и $g_{\perp}$ в выражение для резонансного магнитного поля. При $g_{\perp}>g_{\|}$получаем $B\left(g_{\perp}\right)<B\left(g_{\|}\right)$из-за обратной пропорциональности $g$ и $B$. Моделирование спектров ЭПР в непрерывном режиме (cw) в порошковых образцах можно сделать относительно легко, поскольку интенсивность сигнала пропорциональна количеству центров в выбранном объеме. Важным преимуществом использования высокочастотного диапазона для систем с анизотропным $g$-фактором является возможность разделения в порошковом материале сигналов с разной ориентацией парамагнитного центра в магнитном поле, то есть выделить наборы микрокристаллов одинаково ориентированных в пространстве.

Следует отметить, что иттербий наряду с четным изотопом с нулевым ядерным магнитным моментом $(I=0$, обогащение 69.4\%) содержит изотопы с ненулевыми ядерными моментами: ${ }^{171} \mathrm{Yb}: I=1 / 2,14.4 \%$, ${ }^{173} \mathrm{Yb}: I=5 / 2,16.2 \%$, которые, естественно, характеризуются сверхтонкими (СТ) взаимодействиями. Мы не учитывали эти взаимодействия при симуляции спектров ЭПР, поскольку они вносят вклад на уровне шумов, тем не менее, в кристаллах эти взаимодействия могут быть разрешены. Для ионов с незаполненной $4 f$-оболочкой константы СТ взаимодействий слабо зависят от кристаллической матрицы и, например, для ионов ${ }^{171} \mathrm{Yb}^{3+}$ по литературным данным варьируются в пределах 800-900 $\cdot 10^{-4} \mathrm{~cm}^{-1}$.

На рис. 2 также приведен результат симуляции спектра ЭПР ионов $\mathrm{Ce}^{3+}$ в порошковом материале, при этом видно, что для церия выполняется обратная ситуация по сравнению $\mathrm{Yb}^{3+}$, когда $g_{Z}>g_{X}, g_{Y}$, при этом $g_{X}$ и $g_{Y}$ существенно отличаются друг от друга, поэтому приведена только низкополевая часть симулированного 
спектра. На вставке справа приведены спектры ЭПР для ионов гадолиния $\mathrm{Gd}^{3+}$ в монокристалле YAG: $\mathrm{Ce}, \mathrm{Gd}$, зарегистрированные в нескольких ориентациях магнитного поля относительно осей кристалла, чтобы продемонстрировать совпадение линий ЭПР в кристалле и в керамике, в которой все возможные ориентации будут присутствовать, то есть исследование порошковых материалов демонстрирует результат природного усреднения по всем возможным ориентациям. Гадолиний входит в керамики как неконтролируемая примесь.

Ион гадолиния $\mathrm{Gd}^{3+}$ имеет наполовину заполненную электронную оболочку с конфигурацией $4 f^{7}$. Основной мультиплет ${ }^{8} S_{7 / 2}$ характеризуется отсутствием орбитального момента $(L=0)$ и значения спинового импульса $S=7 / 2$. Восьмикратно вырожденный уровень свободного иона трехвалентного гадолиния, помещенного в кристаллическое поле ромбической симметрии, делится на четыре крамерсовых дублета.

Подобно ионам $\mathrm{Ce}^{3+}$, ионы $\mathrm{Gd}^{3+}$ замещают ионы $\mathrm{Y}^{3+}$ в YAG и $\mathrm{Lu}^{3+}$ в LuAG и занимают додекаэдрические позиции (с-позиции). В кристалле граната имеется шесть магнитно-неэквивалентных положений $\mathrm{Gd}^{3+}$, поэтому при произвольной ориентации кристалла в магнитном поле должна наблюдаться суперпозиция шести спектров ЭПР. Для низких концентраций изолированных ионов $\mathrm{Gd}^{3+}$ в кристаллах с диамагнитной основой спектр ЭПР состоит из семи линий тонкой структуры для каждого из шести магнитно-неэквивалентных центров, которые являются результатом как внутри дублетных, так и междублетных переходов в приближении сильных магнитных полей.

На рис. 2 присутствует сигнал в низких магнитных полях, который, по-видимому, принадлежит некрамерсовым ионам $\mathrm{Tb}^{3+}(S=3)$ с расщеплением тонкой структуры порядка $3 \mathrm{~cm}^{-1}$.

На рис. 3 представлен спектр ЭПР в керамике YAG : Ce,Cr, зарегистрированный в непрерывном режиме на частоте $94 \mathrm{GHz}$ при двух температурах: 1.5 и $5 \mathrm{~K}$. Приведен результат симуляции спектра ЭПР ионов $\mathrm{Ce}^{3+}$ в порошковом материале, повторяющий зависимость, представленную на рис. 2. Также представлена симуляция спектра ЭПР в порошковом материале для ионов хрома $\mathrm{Cr}^{3+}$, имеющего квадруплетное состояние для спина $S=3 / 2$ с аксиальной симметрией и с параметром тонкой структуры $D=7.856 \mathrm{GHz}$. Форма спектра является результатом того факта, что число спиновых пакетов, вносящих вклад в спектр, намного больше в плоскости $x y$, чем вдоль оси $z$. Спектр ЭПР рассчитывается в предположении наличия большого количества парамагнитных центров со случайной ориентацией относительно статического магнитного поля $B$. При симуляции использовались спиновые пакеты с шириной линии $\Delta B=1 \mathrm{mT}$. При повышении температуры $(5 \mathrm{~K})$ спектр ЭПР ионов $\mathrm{Ce}^{3+}$ не виден из-за уменьшения времени спин- решеточной релаксации.

Хром $\mathrm{Cr}^{3+}$ является распространенной примесью в кристаллах и порошках $\mathrm{Y}_{3} \mathrm{Al}_{5} \mathrm{O}_{12}$. Его взаимодействие

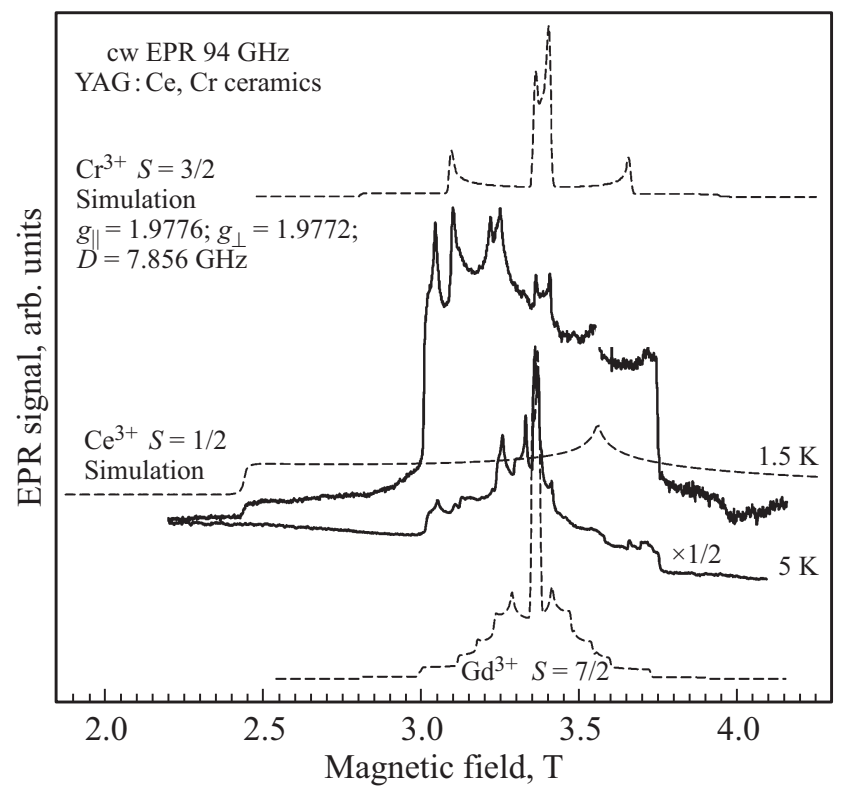

Рис. 3. Спектр ЭПР, зарегистрированный в керамике $\mathrm{YAG}$ : Ce,Cr. Пунктиром показаны результаты симуляции спектров ЭПР ионов $\mathrm{Ce}^{3+}, \mathrm{Cr}^{3+}$ и $\mathrm{Gd}^{3+}$ в порошковом материале.

с собственными дефектами решетки и их влияние на электронную рекомбинацию и компенсацию носителей заряда в YAG широко изучены $[23,24]$.

Хром характеризуется излучением в красном спектральном диапазоне, соответствующем переходу ${ }^{4} T_{2} \rightarrow{ }^{4} A_{2}$ иона $\mathrm{Cr}^{3+}$ в YAG на длине волны $687 \mathrm{~nm}[25,26]$, при этом происходит эффективная передача энергии от возбужденных центров церия к хрому в YAG [27]. Таким образом, совместное легирование люминофоров на основе YAG: Се хромом является перспективным способом увеличения количества красного излучения люминесцентных диодов на основе YAG: Ce. Отметим, что хром является распространенным загрязнением в порошках и монокристаллах YAG: Ce, поэтому контроль хрома важен для применений.

Хром входит в YAG в состоянии $\mathrm{Cr}^{3+}\left(3 d^{3}\right)$ и занимает в основном октаэдрическую позицию алюминия (а-позиция). Спиновый гамильтониан (1) для такого центра упрощается до выражения

$$
\begin{aligned}
H=g_{\|} \mu_{B} H_{z} S_{z} & +g_{\perp} \mu_{B}\left(H_{x} S_{x}+H_{y} S_{y}\right) \\
& +D\left(S_{z}^{2}-1 / 3 S(S+1) .\right.
\end{aligned}
$$

Локальные оси $x, y, z$ обозначают здесь кислородные октаэдры, где ось $z$ выбирается вдоль локальных компонент тригонального кристаллического поля октаэдра (симметрия $C_{3 i}$ ). Значения параметров $g_{\|}=1.9776$, $g_{\perp}=1.9772$ и $D=7.856 \mathrm{GHz}$ для $\mathrm{Cr}^{3+}\left(3 d^{3}\right)$ в $\mathrm{YAG}$ находились в различных работах по ЭПР на кристаллах YAG: $\mathrm{Cr}[28,29,30]$.

На рис. 3 виден усредненный спектр ЭПР ионов $\mathrm{Gd}^{3+}$, гадолиний входит в керамики как неконтролиру- 


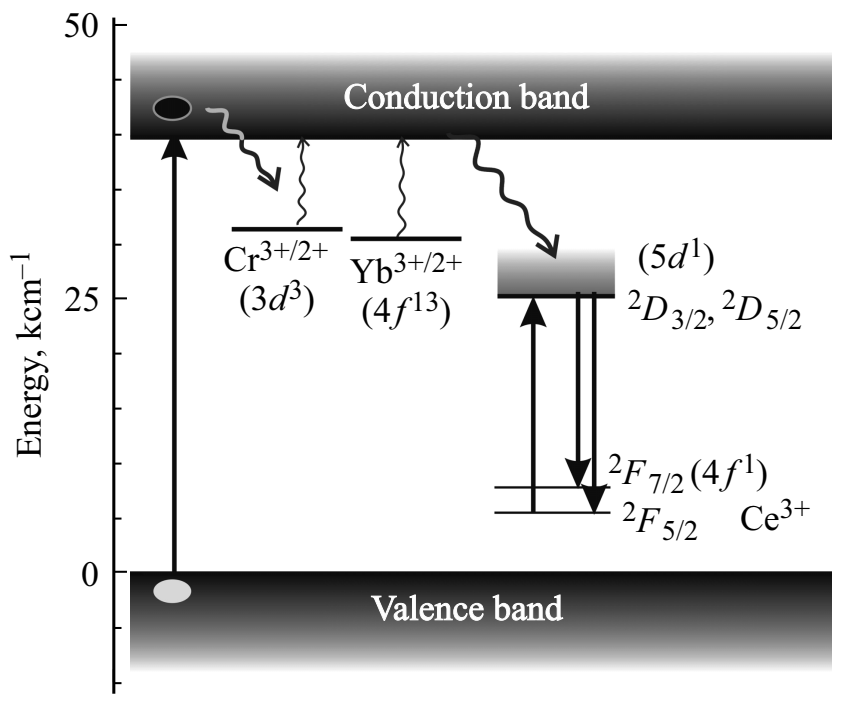

Pис. 4. Диаграмма запрещенной зоны $\mathrm{YAG}: \mathrm{Ce}, \mathrm{Yb}$, показаны глубины залегания тепловых ловушек $\mathrm{Cr}^{3+/ 2+}$ и $\mathrm{Yb}^{3+/ 2+}$ относительно дна зоны проводимости.

емая примесь. Внизу пунктиром приведена симуляция порошкового спектра ЭПР для ионов $\mathrm{Gd}^{3+}, S=7 / 2$, $g=1.991$, использовались параметры для ромбической симметрии кристаллического поля $D=2.275 \mathrm{GHz}$, $E=0.240 \mathrm{GHz}[31]$, остальные параметры спинового гамильтониана, вносящие незначительный вклад в усредненный спектр, не учитывались.

На рис. 4 показана диаграмма запрещенной зоны YAG: $\mathrm{Ce}, \mathrm{Yb}$ и глубины тепловых ловушек $\mathrm{Cr}^{3+/ 2+}$ и $\mathrm{Yb}^{3+/ 2+}$ относительно дна зоны проводимости. Эти данные получены в работе [7] на основе результатов по термостимулированной люминесценции и послесвечению в исследованных керамиках. Видно, что наличие неконтролируемых примесей хрома и иттербия в керамиках гранатов могут существенно изменить динамику излучательных процессов ионов $\mathrm{Ce}^{3+}$, поэтому контроль этих примесей важен для применений этих материалов.

На рис. 5 показаны спектры ЭПР, зарегистрированные на частоте $94 \mathrm{GHz}$ в керамике $\mathrm{Gd}_{2.7} \mathrm{Lu}_{0.3} \mathrm{Ga}_{2.5} \mathrm{Al}_{2.5} \mathrm{O}_{12}: \mathrm{Ce}(0.4 \%)$ при разных температурах. Вверху, серым цветом, для сравнения приведен спектр ЭПР в керамике $\mathrm{c}$ малой концентрацией гадолиния, в которой виден сигнал ЭПР одиночных ионов $\mathrm{Gd}^{3+}$, представленный выше на рис. $2 ; g$-фактору $g=2.00$ соответствует магнитное поле $\approx 3.3 \mathrm{~T}$. Пунктиром показан спектр ЭПР, зарегистрированный по электронному спиновому эху при $1.5 \mathrm{~K}$.

При таком высоком содержании Gd ожидается образование комплексов $\mathrm{Gd}$, включающих несколько ионов $\mathrm{Gd}^{3+}$, связанных спин-спиновым взаимодействием, начиная с парных центров $\mathrm{Gd}^{3+}-\mathrm{Gd}^{3+}$ и заканчивая образованием четверок взаимодействующих ионов $\mathrm{Gd}^{3+}$. Например, для парных центров $\mathrm{Gd}-\mathrm{Gd}$ ЭПР-переходы можно описать спиновым гамильтонианом

$$
H=H_{\mathrm{Gd} 1}+H_{\mathrm{Gd} 2}+S_{1} J S_{2},
$$

где $H_{\mathrm{Gd} 1}$ и $H_{\mathrm{Gd} 2}-$ спиновые гамильтонианы обоих ионов $\mathrm{Gd}^{3+}$ пары, а $J$ - тензор спин-спинового взаимодействия, включающий вклад как диполь-дипольного, так и обменного взаимодействий.

Даже для магнитно-эквивалентных ионов Gd, для которых $g$ и тензоры тонкой структуры имеют те же главные оси, что и тензор спин-спинового взаимодействия, вычисления являются сложными. Для пар, которые взаимодействуют как магнитно-неэквивалентные ионы, ожидаются малые недиагональные члены в спиновом гамильтониане, что еще больше усложняет вычисления. Ранее нами [12] с использованием метода оптически детектируемого магнитного резонанса (ОДМР) значение спин-спинового взаимодействия для пар $\mathrm{Gd}^{3+}-\mathrm{Gd}^{3+}$ было приблизительно оценено как $\sim 1 \mathrm{~cm}^{-1}$, что близко к параметрам спин-спинового взаимодействия пар $\mathrm{Ce}-\mathrm{Ce}$ в YAlO3: Ce.

Линии ЭПР с тонкой структурой сильно анизотропны, поэтому в образцах керамики усреднение по всем возможным ориентациям и всем магнитно-неэквивалентным одиночным $\mathrm{Gd}^{3+}$-центрам и их кластерам приводит к сглаживанию анизотропных линий ЭПР и увеличению интенсивности линий со слабой угловой зависимостью.

Интенсивность сигналов растет при понижении температуры вплоть $1.5 \mathrm{~K}$, что свидетельствует о ферромагнитной природе обменного взаимодействия [32].

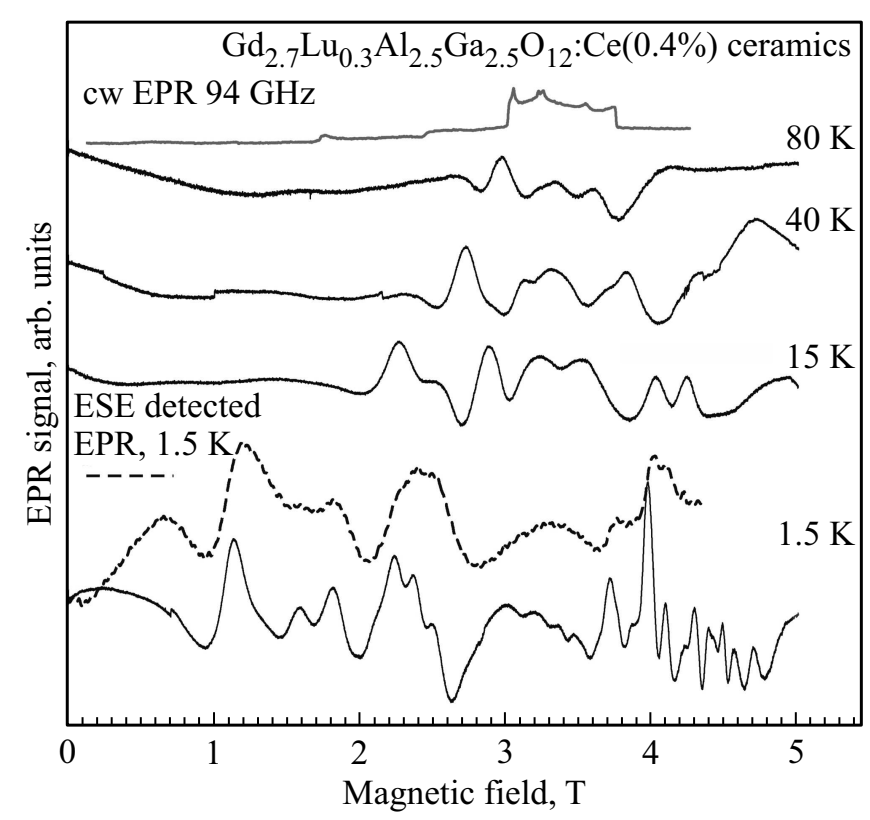

Рис. 5. Спектры ЭПР, зарегистрированные в керамике $\mathrm{Gd}_{2.7} \mathrm{Lu}_{0.3} \mathrm{Ga}_{2.5} \mathrm{Al}_{2.5} \mathrm{O}_{12}: \mathrm{Ce}(0.4 \%)$ при разных температурах. Пунктиром показан спектр ЭПР, зарегистрированный по электронному спиновому эху при $1.5 \mathrm{~K}$. Вверху, серым цветом, приведен спектр ЭПР в керамике с малой концентрацией гадолиния. 


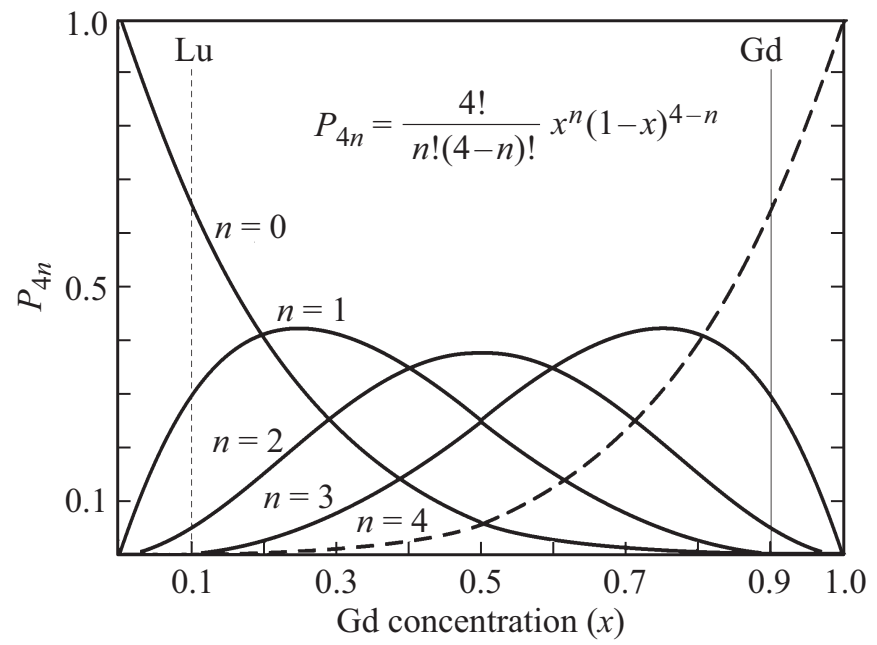

Рис. 6. Графики для концентраций изолированных ионов $\mathrm{Gd}^{3+}$ $(n=0)$, пар $(n=1)$, троек $(n=2)$ и четверок $(n=3)$, в зависимости от содержания гадолиния $(x)$ в кристалле граната.

Обменные кластеры могут содержать как пары одинаковых парамагнитных центров (ионов гадолиния), так и более сложные комбинации этих ионов. Обменные кластеры, содержащие три иона гадолиния, являются простейшими после димеров многоцентровыми обменными структурами, среди которых особое место занимают кластеры, в которых магнитные ионы образуют равносторонний треугольник. Так как существует только один тип ионов $\mathrm{O}^{2-}$, тетраэдрически связанный с двумя ионами $\mathrm{A}^{3+}$ и двумя ионами $\mathrm{Y}^{3+}$, косвенный обмен между ионами гадолиния $\mathrm{Gd}^{3+}$, занимающими позиции иттрия, может осуществляться через соседние ионы кислорода.

Важным результатом является наблюдение электронного спинового эха в таких системах, поскольку это дает возможность исследовать релаксационные процессы в магнитно-разбавленных материалах. Следует отметить, что интенсивность сигналов ЭПР, зарегистрированных по ЭСЭ, на несколько порядков превышает интенсивность сигналов, зарегистрированных в непрерывном режиме.

На рис. 6 приведены графики для концентраций изолированных ионов $\mathrm{Gd}^{3+}(n=0)$, пар $(n=1)$, троек $(n=2)$ и четверок $(n=3)$ в зависимости от содержания гадолиния $(x)$ в кристалле. $P_{4 n}-$ концентрация ионов $\mathrm{Gd}^{3+},\left(\mathrm{Lu}^{3+}\right)$, имеющих $n$ соседних ионов $\mathrm{Gd}^{3+},\left(\mathrm{Lu}^{3+}\right)$ в ближайшей координационной сфере додекаэдрической позиции (с-позиции), $x$ концентрация ионов $\mathrm{Gd}^{3+}$, $\left(\mathrm{Lu}^{3+}\right)$. Для $\mathrm{Gd}^{3+} x=0.9$, для $\mathrm{Lu}^{3+} x=0.1$. Ионы церия $\mathrm{Ce}^{3+}$ не рассматриваются, ввиду их малой концентрации $(x<0.005)$. При расчетах использовалась стандартная формула для расчета вероятностей образования одиночных и сложных центров

$$
P_{4 n}=\frac{4 !}{n !(4-n) !} x^{n}(1-x)^{4-n}
$$

Видно, что для приведенной концентрации гадолиния наиболее вероятно образование троек и четверок ионов гадолиния, причем, для четверок, ввиду целого спина $S=4 \cdot 7 / 2=14$, на основании вида температурных зависимостей интенсивности спектров ЭПР, реализуется ферромагнитное упорядочение спинов ионов $\mathrm{Gd}^{3+}$.

\section{4. Заключение}

В работе продемонстрированы возможности разработанного в ФТИ инновационного ЭПР оборудования для диагностики на электронном уровне примесного состава керамик на основе гранатов путем регистрации спектров ЭПР в высокочастотном микроволновом диапазоне (94 и $130 \mathrm{GHz}$ ) в непрерывном и импульсном режимах с возможностью сканирования магнитного поля в диапазоне $-7-+7 \mathrm{~T}$ с переходом через нулевое магнитное поле, температурный диапазон $1.5-300 \mathrm{~K}$. Были обнаружены и идентифицированы спектры ЭПР примесных ионов церия $\mathrm{Ce}^{3+}$, иттербия $\mathrm{Yb}^{3+}$, хрома $\mathrm{Cr}^{3+}$ и гадолиния $\mathrm{Gd}^{3+}$ в керамиках иттрий алюминиевого граната $\mathrm{Y}_{3} \mathrm{Al}_{5} \mathrm{O}_{12}(\mathrm{YAG})$ на частоте $94 \mathrm{GHz}$. Проведена симуляция анизотропных спектров ЭПР в порошковых материалах путем усреднения этих спектров ЭПР по различным ориентациям. Показано преимущество измерения спектров ЭПР в высокочастотном диапазоне, по сравнению со стандартной техникой ЭПР, позволяющее разделить спектры ЭПР, характеризующиеся разными анизотропными $g$-факторами, а также выделить сигналы ЭПР, обусловленные расщеплением тонкой структуры для центров с высоко-спиновыми состояниями. В керамиках с высоким содержанием магнитных ионов $\mathrm{Gd}^{3+}$ наблюдались спектры ЭПР много-ионных комплексов гадолиния, при этом сделано предположение, что при низких температурах видны спектры ЭПР комплексов с максимальным количеством обменно-связанных ионов гадолиния (четырех ионов) и по мере повышения температуры последовательно видны сигналы от трех-, двухчастичных комплексов и изолированных ионов гадолиния. Исследования температурных зависимостей спектров ЭПР свидетельствуют о ферромагнитном упорядочении обменно-связанных комплексов гадолиния.

\section{Финансирование работы}

Работа выполнена при поддержке Министерства науки и высшего образования, соглашение № 14.604.21.0200, идентификатор проекта: RFMEFI60417X0200.

\section{Конфликт интересов}

Авторы заявляют, что у них нет конфликта интересов. 


\section{Список литературы}

[1] M. Nikl, V.V. Laguta, A. Vedda. Phys. Status Solidi B 245, 1701 (2008).

[2] C. Dujardin, C. Mancini, D. Amans, G. Ledoux, D. Abler, E. Auffray, P. Lecoq, D. Perrodin, K. Ovanesyan, A. Petrosyan. J. Appl. Phys. 108, 013510 (2010).

[3] R. Autrata, P. Schauer, Jos. Kvapil, J. Kvapil. J. Phys. E 11, 707 (1978).

[4] M. Moszynski, T. Ludziewski, D. Wolski, W. Klamra, L.O. Norlin. Nucl. Instrum. Meth. Phys. Sect. A 345, 461 (1994).

[5] P. Schlotter, R. Schmidt, J. Schneider. J. Appl. Phys. A 64, 417 (1997).

[6] V. Bachmann, C. Ronda, A. Meijerink. Chem. Mater. 21, 2077 (2009).

[7] V. Khanin, I. Venevtsev, S. Spoor, J. Boerekamp, A.M. van Dongen, H.Wieczorek, K. Chernenko, D. Buettner, C. Ronda, P. Rodnyi. Opt. Mater. 72, 161 (2017).

[8] R. Kolesov, K. Xia, R. Reuter, M. Jamali, R. Stohr, T. Inal, P. Siyushev, J. Wrachtrup. Phys. Rev. Lett. 111, 120502 (2013).

[9] P. Siyushev, K. Xia, R. Reuter, M. Jamali, N. Zhao, N. Yang, C. Duan, N. Kukharchyk, A. D. Wieck, R. Kolesov, J. Wrachtrup. Nature Commun. 5, 3895 (2014).

[10] K. Xia, R. Kolesov, Ya Wang, P. Siyushev, R. Reuter, T. Kornher, N. Kukharchyk, A.D. Wieck, B. Villa, S. Yang, J. Wrachtrup. Phys. Rev. Lett. 115, 093602 (2015).

[11] A.G. Badalyan, G.V. Mamin, Yu.A. Uspenskaya, E.V. Edinach, H.R. Asatryan, N.G. Romanov, S.B. Orlinskii, P.G. Baranov, V.M. Khanin, H. Wieczorek, C. Ronda. Phys. Status Solidi B 254, 1600631 (2017).

[12] D.O. Tolmachev, A.S. Gurin, Yu.A. Uspenskaya, G.R. Asatryan, A.G. Badalyan, N.G. Romanov, A.G. Petrosyan, P.G. Baranov, H. Wieczorek, C. Ronda. Phys. Rev. B 95, 224414 (2017).

[13] Yu.A. Uspenskaya, G.V. Mamin, R.A. Babunts, A.G. Badalyan, E.V. Edinach, H.R. Asatryan, N.G. Romanov, S.B. Orlinskii, V.M. Khanin, H. Wieczorek, C. Ronda, P.G. Baranov. AIP Adv 8, 035001 (2018).

[14] H.R. Lewis. J. Appl. Phys. 37, 739 (1966).

[15] G.R. Asatryan, D.D. Kramushchenko, Yu.A. Uspenskaya, P.G. Baranov, A.G. Petrosyan. Phys. Solid State 56, 1150 (2014).

[16] G.F. Herrmann, J.J. Pearson, K.A. Wickersheim. J. Appl. Phys. 37, 1312 (1966).

[17] D.L. Wood. J. Chem. Phys. 39, 1671 (1963).

[18] R.A. Buchanan, K.A. Wickersheim, J.J. Pearson, G.F. Herrmann. Phys. Rev. 159, 245 (1967).

[19] M.T. Hutchings, W.P. Wolf. J. Chem. Phys. 41, 617 (1964).

[20] J.J. Pearson, G.F. Herrmann, K.A. Wickersheim, R.A. Buchanan. Phys. Rev. 159, 251 (1967).

[21] D.S. Sumida, T.Y. Fan. OSA Proc. 10, 100 (1994).

[22] J.W. Carson, R.L. White. J. Appl. Phys. 31, S53 (1960).

[23] V.A. Akkerman, G.R. Bulka, D.I. Vainshtein, V.M. Vinokurov, A.A. Galeev, G.A. Ermakov, V.M. Lyubchenko, A.A. Markelov, N.M. Nizamutdinov, N.M. Khasanova. Sov. Phys. Solid State 34, 398 (1992).

[24] I.Sh. Akhmadullin, S.A. Migachev, S.P. Mironov. Nucl. Instrum. Meth. Phys. Res. B 65, 270 (1992).

[25] G. Bums, E.A. Geiss, B.A. Jenkins, M.I. Nathan. Phys. Rev. 139, A1687 (1965).
[26] D.L. Wood, J. Ferguson, K. Knox, J.F. Dillon. J. Chem. Phys. 39, 890 (1963).

[27] G. Blasse, A. Bril. J. Chem. Phys. 47, 5139 (1967).

[28] J.W. Carson, R.L. White. J. Appl. Phys. 32, 1787 (1961).

[29] L.J. Schwee, J.R. Cunningham. J. Appl. Phys. 37, 449 (1966).

[30] R. Valentin, H. Luft, K. Baberschke. Phys. Status Solidi B 48, 763 (1971).

[31] V.A. Vazhenin, A.P. Potapov, G.R. Asatryan, Yu.A. Uspenskaya, A.G. Petrosyan, A.V. Fokin. Phys. Solid State 58, 1627 (2016).

[32] Ю.В. Яблоков, В.К. Воронкова, Л.В. Мосина. Парамагнитный резонанс обменных кластеров. Наука, М. (1988). $181 \mathrm{c.}$

Редактор Т.Н. Василевская 